\title{
Three-dimensional analysis of the surface registration accuracy of electromagnetic navigation systems in live endoscopic sinus surgery*
} \author{
Po-Wen Cheng ${ }^{1,2}$ \\ ' Department of Otolaryngology, Far Eastern Memorial Hospital, Taipei, Taiwan \\ ${ }^{2}$ Oriental Institute of Technology, Taipei, Taiwan
}

Chih-Ming Chang ${ }^{1}$, Kai-Min Fang 1,2, Tsung-Wei Huang 1,2, Chi-Te Wang 1,2,

Rhinology 51: 343-348, 2013 DOl:10.4193/Rhino12.165

*Received for publication:

October 4, 2012

Accepted: April 30, 2013

\section{Summary}

Background: Studies on the performance of surface registration with electromagnetic tracking systems are lacking in both live surgery and the laboratory setting. This study presents the efficiency in time of the system preparation as well as the navigational accuracy of surface registration using electromagnetic tracking systems.

Methodology: Forty patients with bilateral chronic paranasal pansinusitis underwent endoscopic sinus surgery after undergoing sinus computed tomography scans. The surgeries were performed under electromagnetic navigation guidance after the surface registration had been carried out on all of the patients. The intraoperative measurements indicate the time taken for equipment set-up, surface registration and surgical procedure, as well as the degree of navigation error along 3 axes.

Results: The time taken for equipment set-up, surface registration and the surgical procedure was $179 \pm 23$ seconds, $39 \pm 4.8$ seconds and $114 \pm 36$ minutes, respectively. A comparison of the navigation error along the 3 axes showed that the deviation in the medial-lateral direction was significantly less than that in the anterior-posterior and cranial-caudal directions.

Conclusion: The procedures of equipment set-up and surface registration in electromagnetic navigation tracking are efficient, convenient and easy to manipulate. The system accuracy is within the acceptable ranges, especially on the medial-lateral axis.

Key words: surface registration, electromagnetic navigation system, endoscopic sinus surgery, computer aided surgery, navigation error

\section{Introduction}

Endoscopic sinus surgery (ESS) for the treatment of sinus disease has been a common otorhinolaryngological surgical procedure since the mid-1980s, with an expanding role in the management of orbital, facial bone and skull base diseases ${ }^{(1)}$. Computer-aided surgery (CAS) technology has been developed to assist surgeons in achieving better anatomical localization since the 1980s $(2,3)$, and may help prevent potential sinus complications such as orbital damage, cerebrospinal fluid leakage and carotid artery injury ${ }^{(4)}$. Based on real-time image guidance in association with endoscopy, CAS systems may help to improve the intraoperative precision of orientation ${ }^{(5,6)}$ using either MRI or CT imaging ${ }^{(7)}$.
Among the commercially available CAS systems, both optical and electromagnetic tracking systems have become popular, as they are more accurate and convenient than electromechanical or sonic tracking systems. The optical tracking system was developed earlier than the electromagnetic system and was the first to be widely adopted in the clinical setting ${ }^{(1)}$. Numerous studies have analyzed its performance under both clinical and laboratory conditions. Conversely, the development of electromagnetic tracking systems for surgical use was limited by ferromagnetic distortion that adversely affected system accuracy until certain hardware and software advances were recently reported ${ }^{(8,9)}$. Currently, the electromagnetic system is much more popular in 
North America.

Navigation accuracy is highly dependent on the registration process, which establishes the correlation between specific landmarks and stored image data. Various registration methods have been proposed, based on external fiducial markers, anatomical landmarks and contour-based registration, respectively. The surface registration procedure that aligns the unique facial contours eliminates the long preparation time of the usual registration technique and is thus clinically convenient ${ }^{(10,11)}$. Thus far, the accuracy of surface registration for optical tracking systems has been shown to be satisfactory in a variety of studies ${ }^{(10-12)}$. Nevertheless, the data for electromagnetic systems using surface registration is lacking for both live surgery and cases of cadaveric dissection. Accordingly, the precision of the surface registration used in electromagnetic tracking systems needs to be further evaluated. We therefore present this study in which we investigate the efficiency of system preparation and the threedimensional accuracy of the surface registration used in electromagnetic tracking systems in live endoscopic sinus surgery.

\section{Materials and methods}

\section{Patients}

Forty patients ( 20 men and 20 women) with chronic paranasal pansinusitis were enrolled in this study between July and December 2011. The severity of disease was classified according to the Lund-Mackay endoscopic grade and CT scan classification systems of chronic rhinosinusitis. All patients were scored as Grade III-IV nasal polyposis according to the endoscopic grade system proposed by Meltzer et al. ${ }^{(13)}$. The total score was more than 15 in the Lund-Mackay CT scan classification system. We excluded patients younger than 18 years, patients with concomitant external sinus surgery, unusual soft tissue sensitivity or damaged tissue at the intended mounting area. We also excluded those with any circulatory disease of the skin that might make it prone to damage by pressure from the silicone contact pad of the head frame used as the navigation reference. Prior to surgery, physicians performed CT scans of the sinus area to obtain images at a $0.75 \mathrm{~mm}$ slice thickness. The image data were then transferred to the navigation unit using a compact disc. The same medical team performed bilateral ESS using the Medtronic S7 navigation system (Medtronic Navigation, Minneapolis, MN, USA) on all patients. The intraoperative measurements in this study indicate the time taken for equipment set-up, surface registration, and surgical procedure, as well as the navigation errors (NEs) along the 3 axes.

\section{Equipment set-up}

After the induction of general anesthesia, a head frame was attached to the patient's forehead using an elastic strap. The head frame was equipped with a headset patient tracker to provide a continuous point of reference for the navigation system. The head frame was placed at the center of the forehead (20-50 $\mathrm{mm}$ above the supraorbital foramen nerve), and was manipulated gently to ensure that it was attached securely and that its position relative to the head would be maintained throughout the course of the surgical procedure. A low-energy electromagnetic field emitter was fixed on a holder and attached to the operating table bed frame (Figure 1). The emitter holder was positioned at least $20 \mathrm{~cm}$ above the operating table and pointed directly at the patient's nose from a distance of $20 \mathrm{~cm}$. No part of the holder was extended into the emitter's electromagnetic field. All of the instrument tracker cables for intraoperative navigation were connected to the navigation unit ports. The time for equipment set-up was recorded for all of the steps.

\section{Surface registration}

Synergy Cranial Software (Medtronic, Minneapolis, MN, USA) was employed for both registration and measurement. Surface registration method was used in this study. The points that were used for the surface scanning were distributed at various locations along the nose, forehead and orbital rim. After collecting a sufficient number of points on the scanned area for computer calculation, the three-dimensional location information was automatically matched with the $\mathrm{CT}$ image. After completing the registration procedure, the correlation between the position of the instrument in the surgical field and the corresponding location on the CT images was established to allow real-time orientation during surgery. No additional registration processes were needed during the operation to adjust for anatomical drift. The time taken for surface registration was recorded.

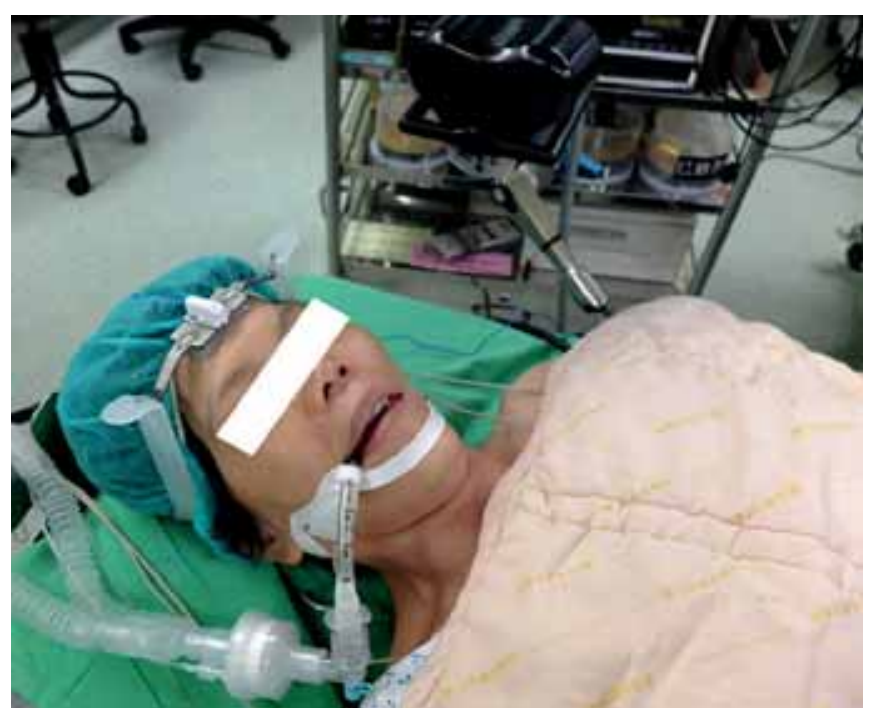

Figure 1. The set-up of the electromagnetic navigation system in endoscopic sinus surgery. 


\section{Navigation error}

During the operation, the patient's head as well as the operating table may sometimes be moved or tilted, and bulb press testing was performed to check the integrity of the lamina papyracea. However, the reference position of the head-tracking system on the forehead must remain unchanged to ensure an accurate navigational reading. We cleared the abnormal sinonasal tissue on both sides to achieve adequate exposure of the skull base, lamina papyracea and sphenoid sinus. A navigational probe was then used to determine the NE. The probe was used to touch a predetermined landmark in the patient. The NE was measured as the vertical distance from the navigated tip of the probe (i.e. the crosshairs) to an imaginary line passing the predetermined CT landmark and perpendicular to the measuring axis (Figure 2) $(12,14,15)$. The NE in the anterior-posterior (AP) direction was measured on the axial plane of the CT scan relative to the nasopharynx (NP) and opening of the sphenoid sinus (OS) reference points, whereas the NE in the cranial-caudal (CC) direction was measured on the sagittal plane using the central points of the anterior and posterior ethmoid roofs (AER, PER, respectively). The NE in the medial-lateral (ML) direction was measured on the coronal plane with the insertion point of the superior turbinate (ST) and the most medial point of the lamina papyracea (LP). For all patients, the measurements of the distance were performed 3 times without repeating the registration process. The mean of these values was considered the NE for specific locations. All the NE results were expressed as absolute values, and those on both sides were averaged for the purposes of statistical analysis.

\section{Statistical methods}

The time of equipment set-up, surface registration and surgical procedure, as well as the NE in the AP, CC and ML directions, are expressed as mean values \pm standard deviations. One-way ANOVA followed by the Bonferroni test was employed for multiple comparison tests. The level of significance was set at $p$ $<0.05$. The statistical package SPSS 16.0 for Windows (SPSS Inc., Chicago, IL, USA) was used for all statistical analyses.

\section{Ethical considerations}

This study was approved by the institutional review board of Far Eastern Memorial Hospital, and written informed consent was obtained from all of the patients.

\section{Results}

Forty patients aged between 24 and 75 years (mean 51.3) underwent bilateral endoscopic sinus surgery. The surgery was primary for 34 patients ( $85 \%$ ) and a revisionary procedure for 6 (15\%). The mean score on the Lund-Mackay CT scan classification system for all of the patients was 19.5 points. The time for the equipment set-up, surface registration and surgical procedure ranged between 141 and $212 \mathrm{~s}$ (mean \pm standard deviation:

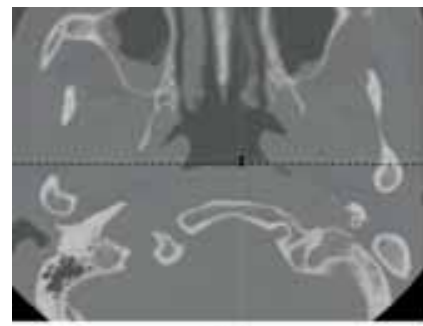

(a)

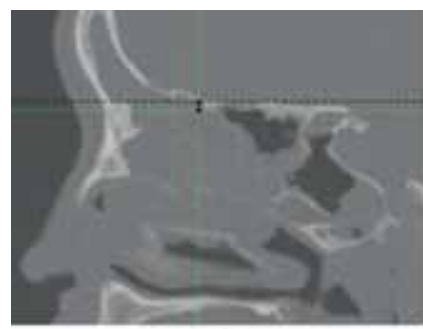

AER

(b)

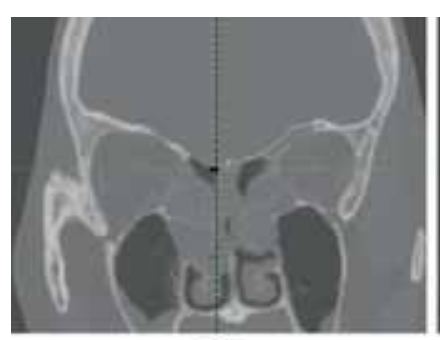

(c)

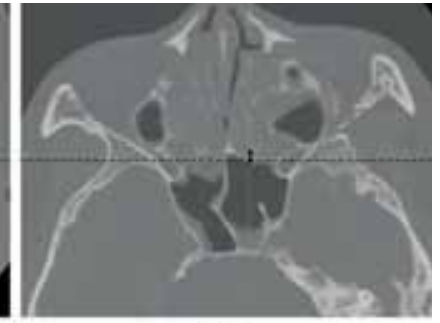

OS

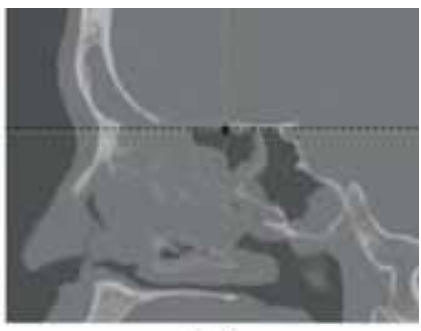

PER

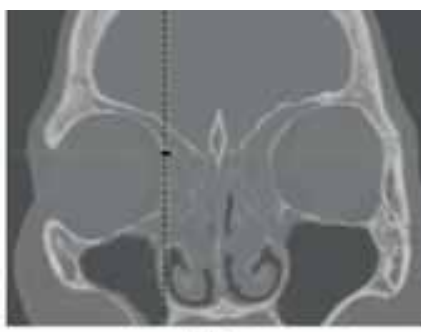

LP
Figure 2. The measurements of the navigation errors at (a) the nasophar$y n x(N P)$ and opening of the sphenoid sinus (OS), (b) the central points of the anterior and posterior ethmoidal roofs (AER, PER, respectively) and (c) the insertion point of the superior turbinate (ST) and the most medial point of the lamina papyracea (LP). Dotted lines indicate imaginary lines passing the predetermined CT landmarks and perpendicular to the measuring axes.

$179 \pm 23), 30$ and $50 \mathrm{~s}(39 \pm 4.8)$, and 60 and $190 \mathrm{~min}(114 \pm 36)$, respectively. In other words, the equipment set-up and surface registration, respectively, took $2.6 \%$ and $0.6 \%$ of the entire procedure time. There were no cases of operative complications.

The NE of the NP, OS, AER, PER, ST and LP was $1.7 \pm 0.9,1.7 \pm 0.9$, $2.1 \pm 1.0,1.7 \pm 1.2,1.0 \pm 0.7$ and $0.8 \pm 0.6 \mathrm{~mm}$, respectively, with a significant difference for these 6 groups ( $p<0.05$, one-way ANOVA test, Figure 3). In comparison to the NE for the 6 location groups, the distance deviation in the ST and LP groups was significantly less than that in the other groups $(p<0.05$, Bonferroni test, Figure 3). No significant differences were observed in any paired comparison between the NP, OS, AER and PER groups ( $p$ $>0.05$, Bonferroni test, Figure 3). Furthermore, there was also no significant difference between the NE in the ST and LP groups ( $p$ $>0.05$, Bonferroni test, Figure 3). 


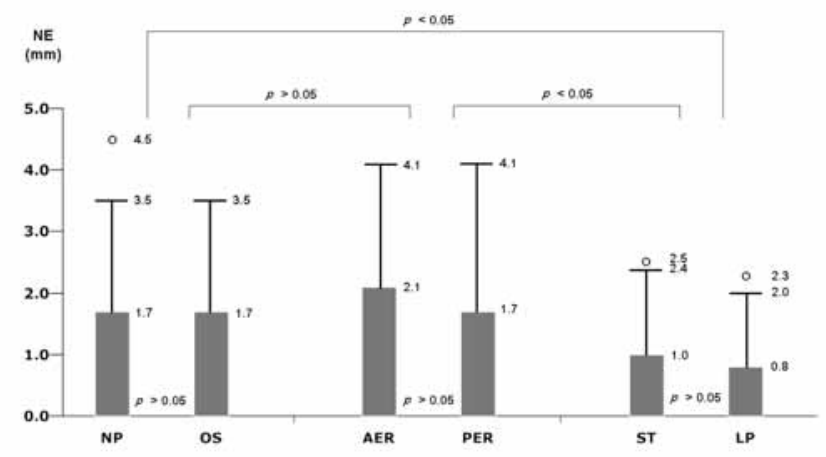

Figure 3. The navigation error (NE) in the 6 location groups. The NE in ST and LP groups (medial-lateral axis) was significantly less than that in the other 4 location groups (anterior-posterior and cranial-caudal axes). Open circles and bars represent the maximum values and upper limits of $95 \%$ confidence intervals, respectively. Top edges of the boxes indicate mean values.

\section{Discussion}

CAS performed by means of image-guided navigation systems has been widely used in sinus and skull base surgeries over the past decade to improve intraoperative orientation, reduce intraoperative complications and enable more precise surgical removal of diseased tissue. The navigation system displays real-time positional information for each tracked instrument on preoperatively acquired images after the registration process, thereby improving an understanding of the patient's anatomy and improving the surgeon's awareness. Surgeons can avoid accidental damage to vital structures in proximity to surgical fields by referencing an exact anatomical orientation, especially in cases in which there is anatomical complexity or intraoperative heavy bleeding ${ }^{(5,12,16)}$. Currently, optical tracking systems are popular because of their availability and relative accuracy ${ }^{(17,18)}$. They use active light-emitting diodes or passive reflecting spheres that are attached to the tracked instruments and reference points on the patient's head so as to localize the position following triangulation using an infrared camera system. Numerous studies have shown that these systems achieve superior spatial resolution and accuracy compared to electromagnetic tracking systems ${ }^{(19,20)}$. However, the direct line of sight between the navigation instrument and infrared camera system may be impeded by the surgeon or instruments, thereby interrupting its ongoing use during the course of an operation, especially in a crowded operative field. Electromagnetic tracking can solve this problem, because trackers composed of coil arrays can detect changes in the electromagnetic field while moving. The instrument's position can be further localized after calculating the relationship between the surgical anatomy and preoperative imaging information. Moreover, electromagnetic tracking systems have improved the accuracy by means of advances in technology and have become more popular in clinical use ${ }^{(8)}$. Nowadays, optical and electromagnetic tracking systems have both come to be accepted as suitable for CAS $(1,18,21)$.

Kral et al. ${ }^{(20)}$ reported that the mean NE of electromagnetic tracking systems in human cadavers was $0.37 \mathrm{~mm}$, which was more precise than the results in this study $(0.8-2.1 \mathrm{~mm})$. This may be explained by the use of externally fixed fiducial markers for paired-point matching registration and immediate measurement following registration in the previous study. Furthermore, unlike cadavers, intraoperative stretching of the skin/soft tissue may occur easily and result in a displaced reference frame, which appears to have been the case in our study ${ }^{(18)}$. The fixation of an endotracheal tube on the patient's face with adhesive tape may also have contributed to the distortion of the facial contours and caused surface registration discordance in the preoperative CT images. All of these factors influence the navigational accuracy during surgery. In this study, we measured the NE during the course of surgical procedures on patients diagnosed with severe sinus diseases. These patients were suitable candidates for navigation surgery and fit the recommendation for CAS proposed by the American Academy of Otolaryngology-Head and Neck Surgery. In contrast to the optimized conditions of laboratory settings, in clinical practice, the conditions tend to reduce system accuracy, especially when long periods have elapsed since surface registration. It may be appropriate to repeat surface registration in consideration of intraoperative anatomical drift to overcome the increased NE in longer operations ${ }^{(6,22)}$. However, we performed surface registration just once, at the beginning of surgery, and defined the NE value intraoperatively to evaluate whether it remained acceptable at the time the ESS was completed.

Although surface registration has been shown to be less accurate than the other registration methods in cadaveric heads, the errors are still within that which is typically deemed the acceptable ranges for navigation ${ }^{(12,23)}$. To our knowledge, this is the first study to measure the NE of the surface registration obtained with an electromagnetic system in live surgery. The results show that the NEs in the 6 location groups have a maximal mean value of $2.1 \mathrm{~mm}$; such variation may be important to surgeons if they, for example, want to assess the anatomical position in relation to the optic nerve or carotid artery. Although there is general agreement that the navigation system may be used for clinical purposes when the error is less than $3 \mathrm{~mm}$ $(24,25)$, the acceptable range of the error may still depend on the location at which the operation is being carried out. Regarding the additional time (mean $218 \mathrm{~s}$ ) for equipment set-up and surface registration, only a small proportion (3.2\%) of the total operation time was spent on system preparation. That is, the extra procedures of preparation for navigation surgery are not time-consuming. 
There are limitations to this study. The time associated with navigation was investigated only in terms of surface registration, and we did not compare different methods of registration in the electromagnetic system, or even compare the electromagnetic system with an optical one. Ecke et al. (1) did evaluate the practicality of navigation systems using the registration of external fiducial makers, with the average preparation time being $15 \pm$ 4.2 minutes and the error being $1.9 \pm 0.3 \mathrm{~mm}$ in the electromagnetic system. Additionally, the optic system required $37 \pm 8.3$ minutes for preparation and had an error of $2.0 \pm 0.6 \mathrm{~mm}$. Compared to a previous report ${ }^{(1)}$, the electromagnetic system using surface registration in this study required a shorter preparation time and yet had a similar number of NEs. Although this study did not compare different registration methods or navigation systems directly, our results, together with the reports in the existing literature ${ }^{(1,10-12)}$, provide information that could be crucial for a surgeon or institution in the selection of a navigation system. Further comparative studies are necessary to investigate the inherent time constraints and errors in the various systems. Furthermore, our results show that the NE in the ML direction had a significantly higher accuracy than measurements in the AP and CC directions. A possible explanation is that the more dispersed registration points resulted in a more accurate anatomical localization. Because the extent of the divergence in tracing the paths is greater in the ML direction than in the AP and CC directions, the NE data consequently show the reading to be more precise on the ML axis. In addition, there is the possibility that depressed skin/soft tissue during the course of surface registration may shift the surface registration points and subsequently result in erroneous positional information, especially in the AP direction.

Most importantly, before CAS may be used with confidence in sinus surgery, the surgeon has to keep in mind that the NEs in any of the axes may affect his or her judgment. As the dissection approaches an important anatomical structure, the users should be aware that the navigational instrument may have touched the critical site, in which case its location may not be correctly displayed on the monitor. Because the navigational accuracy is less reliable in the AP and CC directions than in the ML direction, dissections near the skull base are of greater risk than those closer to the lamina papyracea. In addition, sinus surgeons in training tend to have an excessive level of trust in the navigation system ${ }^{(26)}$; such an excessive reliance on the navigation system can lead to a neglect of personal anatomical knowledge on the part of the surgeon and result in needless surgical complications. Although substantial, but acceptable NE levels were confirmed in this study, it emphasized that CAS is only a useful adjunct to surgical experience and anatomical knowledge when it is applied properly ${ }^{(26)}$.

In conclusion, electromagnetic navigation systems using surface registration are a suitable adjuvant tool for ESS. It takes less than 4 minutes to complete the equipment set-up and surface registration, and it offers invaluable real-time information on location with sufficient accuracy. In the three-dimensional analysis of surface registration accuracy, the deviations in the ML direction are significantly less than those for the other two axes. This finding suggests that surgeons should be more cautious when dissecting in the AP or CC direction during the course of navigation for sinus surgery.

\section{Acknowledgement}

This study was supported by grants of Far Eastern Memorial Hospital (FEMH-2013-C-041), Taiwan.

\section{Authorship contribution}

CMC: wrote the paper; KMF, TWH, CTW: analysed and interpreted data; PWC: performed surgeries and supervised the study.

\section{Conflict of interest}

The authors have declared that they have no conflict of interest.

\section{References}

1. Ecke U, Luebben B, Maurer J, Boor S, Mann WJ. Comparison of Different ComputerAided Surgery Systems in Skull Base Surgery. Skull Base. 2003; 13: 43-50.

2. Schloendorff G, Moesges R, Meyer-Ebrecht D, Krybus W, Adams L. CAS (computer assisted surgery). A new procedure in head and neck surgery. HNO. 1989; 37: 187-190.

3. Anon JB. Computer-aided endoscopic sinus surgery. Laryngoscope. 1998; 108: 949-961.

4. Mafee MF, Chow JM, Meyers R. Functional endoscopic sinus surgery: anatomy, CT screening, indications, and complications. AJR Am J Roentgenol. 1993; 160: 735-744.

5. Hepworth EJ, Bucknor M, Patel A, Vaughan
WC. Nationwide survey on the use of image-guided functional endoscopic sinus surgery. Otolaryngol Head Neck Surg. 2006; 135: 68-73.

6. Al-Swiahb JN, Al Dousary SH. Computeraided endoscopic sinus surgery: a retrospective comparative study. Ann Saudi Med 2010; 30: 149-152

7. Fried MP, Topulos G, Hsu L, et al. Endoscopic sinus surgery with magnetic resonance imaging guidance: initial patient experience. Otolaryngol Head Neck Surg. 1998; 119: 374-380.

8. Hummel J, Figl M, Birkfellner W, et al. Evaluation of a new electromagnetic tracking system using a standardized assess- ment protocol. Phys Med Biol. 2006; 51: N205-210.

9. Yaniv Z, Wilson E, Lindisch D, Cleary K. Electromagnetic tracking in the clinical environment. Med Phys. 2009; 36: 876-892.

10. Stelter K, Andratschke M, Leunig A, Hagedorn H. Computer-assisted surgery of the paranasal sinuses: technical and clinical experience with 368 patients, using the Vector Vision Compact system. J Laryngol Otol. 2006; 120: 1026-32.

11. Schlaier J, Warnat J, Brawanski A. Registration accuracy and practicability of laser-directed surface matching. Comput Aided Surg 2002; 7: 284-290.

12. Ledderose GJ, Stelter K, Leunig A, Hagedorn 
H. Surface laser registration in ENT-surgery: accuracy in the paranasal sinuses--a cadaveric study. Rhinology. 2007; 45: 281-285.

13. Meltzer EO, Hamilos DL, Hadley JA, et al. Rhinosinusitis: Developing guidance for clinical trials. Otolaryngol Head Neck Surg. 2006; 135: S31-80.

14. Fitzpatrick JM, West JB, Maurer CR Jr. Predicting error in rigid-body point-based registration. IEEE Trans Med Imaging. 1997; 17: 694-702.

15. Labadie RF, Davis BM, Fitzpatrick JM Image-guided surgery: what is the accuracy? Curr Opin Otolaryngol Head Neck Surg. 2005; 13: 27-31.

16. Metson RB, Cosenza MJ, Cunningham MJ, Randolph GW. Physician experience with an optical image guidance system for sinus surgery. Laryngoscope. 2000; 110: 972-976.

17. Grevers G, Menauer F, Leunig A Caversaccio M, Kastenbauer E. Navigation surgery in diseases of the paranasal sinuses. Laryngorhinootologie. 1999; 78: 41-46.

18. Klimek L, Ecke U, Lübben B, Witte J, Mann W. A passive-marker-based optical system for computer-aided surgery in otorhinolaryngology: development and first clinical experiences. Laryngoscope. 1999; 109: 1509-1515.
19. Schmerber S, Chassat F. Accuracy evaluation of a CAS system: laboratory protocol and results with $6 \mathrm{D}$ localizers, and clinical experiences in otorhinolaryngology. Comput Aided Surg. 2001; 6: 1-13.

20. Kral F, Puschban EJ, Riechelmann H, Pedross F, Freysinger W. Optical and electromagnetic tracking for navigated surgery of the sinuses and frontal skull base. Rhinology 2011; 49: 364-368.

21. Fried MP, Kleefield J, Gopal H, Reardon E, Ho BT, Kuhn FA. Image-guided endoscopic surgery: results of accuracy and performance in a multicenter clinical study using an electromagnetic tracking system. Laryngoscope. 1997; 107: 594-601.

22. Metson R, Gliklich RE, Cosenza M. A comparison of image guidance systems for sinus surgery. Laryngoscope. 1998; 108: 1164-1170.

23. Knott PD, Batra PS, Butler RS, Citardi MJ. Contour and paired-point registration in a model for image-guided surgery. Laryngoscope. 2006; 116: 1877-1881.

24. Metson RB. Image-guided sinus surgery: Lessons learnt from the first 1000 cases. Otolaryngol Head Neck Surg. 2003; 128: 8-13.

25. Roth M, Lanza DC, Zinreich J, Yousem D, Scanlan KA, Kennedy DW. Advantages and disadvantages of three-dimensional computed tomography intraoperative localization for functional endoscopic sinus surgery. Laryngoscope. 1995; 105: 1279-1286.

26. Stelter K, Ertl-Wagner B, Luz M, et al. Evaluation of an image-guided navigation system in the training of functional endoscopic sinus surgeons. A prospective, randomised clinical study. Rhinology. 2011; 49: 429-437.

Po-Wen Cheng, MD

Department of Otolaryngology

Far Eastern Memorial Hospital

Taipei, Taiwan

21, Sec.2, Nanya S. Rd, Banqiao Dist.

New Taipei City

Taiwan

Tel: + 886-2-89667000 ext 2833

Fax: + 886-2-77282149

E-mail: powenjapan@yahoo.com.tw 